\title{
COLEÇÃO DE ABELHAS COMO FERRAMENTA DIDÁTICA FACILITADORA PARA A APRENDIZAGEM NO ENSINO TÉCNICO
}

\author{
P.D.C. MOURA ${ }^{1}$, M.S. BEZERRA ${ }^{2}$, D.F. BRASIL ${ }^{3}$, M.O.GUIMARÃES-BRASIL ${ }^{4}$ \\ Instituto Federal de Educação, Ciência e Tecnologia do Rio Grande do Norte \\ Universidade Federal Rural do Semiárido ${ }^{3}$ \\ danieldfb@gmail.com ${ }^{3}$
}

Submetido 14/03/2019 - Aceito 17/02/2020

DOI: $10.15628 /$ holos. 2020.8398

\section{RESUMO}

Com o passar dos tempos, novos estudos sobre materiais didáticos vêm sendo desenvolvidos, com a intenção de aumentar a interação na sala de aula, proporcionando maior conforto e capacidade de assimilação dos alunos, além de desenvolver suas capacidades cognitivas. Neste sentido, este trabalho tem por objetivo analisar a eficácia de um instrumento didático, qual seja, uma coleção de abelhas, a partir de uma pesquisa desenvolvida com a utilização de questionários aplicados antes e depois da construção e utilização dessa coleção, tendo como público de pesquisa as turmas de primeiro ano do curso Técnico em Apicultura. A partir disso, os resultados mostraram a eficiência de uma coleção de abelhas como ferramenta mediadora no processo de ensino e aprendizagem. Quando avaliado o aumento dos percentuais estatísticos, conclui-se que a montagem e utilização da coleção de abelhas auxiliou na aprendizagem e desenvolvimento dos alunos, demonstrando que a criação de novas ferramentas didáticas são essenciais para a educação técnica.

PALAVRAS-CHAVE: apicultura, educação, entomologia, questionário

\section{BEE COLLECTION AS A FACILITATING TOOL FOR LEARNING IN TECHNICAL EDUCATION}

\begin{abstract}
Over time new studies on teaching materials have been developed, with the intention of increasing interaction in the classroom, providing greater comfort and capacity for assimilation of students in addition to developing cognitive abilities. In this sense, this work has the objective of analyzing the effectiveness of a didactic instrument based on a research developed with the use of questionnaires applied before and after the construction and use of a collection of bees, having as
\end{abstract}

research public the first year classes of the Beekeeping Technician course. From this, the results showed the efficiency of a bee collection as a mediating tool in the teaching and learning process. When evaluating the increase in statistical percentages, it was concluded that the assembly and use of the collection of bees aided in the learning and development of the students, demonstrating that the creation of new didactic tools is essential for technical education.

KEYWORDS: beekeeping, education, entomology, questionnaire 


\section{INTRODUÇÃO}

A utilização de recursos didáticos não convencionais como alternativa ao ensino vem contribuindo para o aumento do nível de interação e aprendizagem, substituindo paulatinamente as tradições livrescas. Uma das alternativas mais viáveis para a substituição dos paradigmas didáticos é a utilização de laboratórios e materiais práticos diversos, que além de incorporar outras dimensões ao ensino, favorecem e ampliam a relação entre alunos e professores (ALBUQUERQUE et al., 2014; GUIMARÃES-BRASIL et al, 2017). Sendo assim, é necessário que o docente venha a intermediar relações entre o discente e o material didático prático a fim de extrair um maior aproveitamento das aulas ministradas (ATAIDE; SILVA, 2011).

Dentre estes materiais incomuns, encontramos a coleção entomológica, utilizada principalmente em demonstrações para alunos e profissionais que estudam os insetos e suas relações ecológicas. A execução e produção destas caixas utilizadas para depósito e manuseio dos insetos sempre desperta um maior interesse dos estudantes (SILVA, 2018). É uma ferramenta altamente acessível devido ao baixo custo de produção, que além de prender a atenção dos alunos devido ao seu elevado potencial de atratividade (SANTOS; SOUTO, 2011), contribui para a desconstrução de paradigmas maléficos associados à maioria dos insetos (SILVA, 2018).

Além disso, a coleção entomológica pode atuar facilitando a aprendizagem tendo em vista que a partir de sua utilização torna-se possível apresentar de maneira tátil a morfologia dos insetos, possibilitando a interação entre as capacidades motoras e sensoria is dos alunos (GULLAN; CRANSTON, 2010). Os insetos, em coleções, representam um grande acervo científico, sendo de suma importância para o estudo da biodiversidade e biogeografia regional (SOUSA-LOPES, 2017).

Dentre os insetos que sempre estão presentes nas caixas entomológicas, estão as abelhas, um dos mais importantes animais da natureza e que estão presentes em quase todos os biomas terrestres, desempenhando papel fundamental para a manutenção e perpetuação dos ecossistemas através da polinização. Essa classe apresenta grande diversidade de espécies e o seu estudo e catalogação dependem de técnicas específicas de coleta e transporte. Estas técnicas permitem a criação de coleções entomológicas, fonte de consultas, observações e pesquisas científicas (CAMARGO et al., 2015).

A confecção de uma coleção entomológica de qualidade é fundamental para pesquisas que visam a identificação precisa das espécies. Dessa maneira, é preciso que os insetos que irão ser estudados sejam capturados e catalogados corretamente, viabilizando assim os mais variados tipos de pesquisa. As coleções de insetos em geral ou de grupos específicos como os das abelhas podem ser utilizadas como materiais de pesquisas por universidades e instituições técnicas, exercendo fundamental papel na construção de conhecimento técnico e profissional, além de facilitar a identificação e diferenciação dos indivíduos coletados (CAMARGO et al., 2015; BEZERRA; MAUES, 2017).

A utilização de recursos pedagógicos práticos complementa o conhecimento adquirido em sala de aula. Neste sentido, este trabalho tem por objetivo analisar a eficácia de um instrumento didático a partir de uma pesquisa desenvolvida com a utilização de questionários aplicados antes 
e depois da construção e utilização de uma coleção de abelhas, tendo como público de pesquisa as turmas de primeiro ano do curso Técnico em Apicultura.

\section{MATERIAL E MÉTODOS}

\subsection{Local da pesquisa}

A pesquisa foi desenvolvida no município de Pau dos Ferros/RN, região do Alto Oeste Potiguar, com estudantes ingressantes no Curso Técnico Integrado em Apicultura, do Instituto Federal de Educação Ciência e Tecnologia do Rio Grande do Norte, durante os meses de abril a julho de 2018. As atividades foram realizadas na disciplina de Biologia das Abelhas, abrangendo 68 alunos. $\mathrm{O}$ quantitativo de estudantes corresponde à amostra avaliada.

\subsection{Descrição da metodologia}

Os dados foram obtidos mediante a construção e aplicação de questionários semiestruturados contendo 26 (vinte e seis) questões, sendo 24 (vinte e quatro) objetivas, uma subjetiva que solicitava a identificação da morfologia da abelha Apis mellifera (antenas, cabeça, tórax e abdômen) e uma questão com apresentação de imagens ilustrativas de abelhas, moscas e vespas para que os alunos destacassem quais eram abelhas. O questionário proposto continha perguntas sobre anatomia e fisiologia das abelhas, noções básicas sobre vida e comportamento das abelhas e diferenças básicas destas com outros insetos, conforme metodologia adaptada de SANTOS E SOUTO (2011).

As aplicações dos questionários aconteceram em dois momentos distintos. O primeiro momento foi realizado no início da disciplina, antes que os alunos tivessem contato com aulas teóricas e práticas. Logo após esta etapa, foram ministradas duas aulas expositivas dialogadas por semana, durante o período de três meses, em que eram abordados conteúdos relacionados à biologia da abelha bem como a construção de uma coleção entomológica de abelhas pelos alunos. Para a confecção da coleção, os alunos foram instruídos a coletar em campo diferentes espécies de abelhas, montá-las em alfinetes entomológicos, secá-las em estufa, etiquetá-las devidamente e organizar todo o material coletado em caixas entomológicas de madeira.

Ao final desta etapa, o mesmo questionário foi reaplicado, para que fosse possível fazer a comparação entre os resultados coletados antes das aulas com o instrumento didático e após as aulas ministradas com o auxílio da ferramenta didática. As respostas dos alunos não foram utilizadas como critério avaliativo da disciplina e não houve nenhuma atribuição de nota por participação.

\subsection{Análise estatística}

Para que os dados fossem analisados com melhor exatidão, os resultados amostrais foram submetidos ao teste de hipóteses, onde a hipótese nula $(\mathrm{HO})$ se refere a igualdade estatística entre a quantidade média de questões assinaladas corretamente nas duas avaliações, e a hipótese alternativa $(\mathrm{H} 1)$ indica se há diferença significativa entre a média do número de questões marcadas de forma correta. A análise estatística se deu através do teste t-Student, visando comparar a média 
de assertivas nos dois questionários. O nível de significância foi estabelecido em $95 \%(p<0,05)$ com n-1 graus de liberdade. As análises estatísticas e a construção dos gráficos foram realizados através dos softwares Microsoft Excel 2016 e Minitab 18.1 (2018).

\section{RESULTADOS E DISCUSSÕES}

A partir dos dados obtidos com as aplicações dos questionários e representados na Figura 1, foi perceptível o aumento no número de acertos no segundo questionário quando comparado ao primeiro, mostrando que a utilização da coleção entomológica, como ferramenta de ensino, pode ser uma prática didática que desperta o interesse e a curiosidade dos alunos (SILVA, 2018; GUIMARÃES-BRASIL, 2017; PEREIRA et al., 2016).



Figura 1: Comparação entre a aplicação do questionário que antecede (linha preta sólida) a utilização da coleção de abelhas e a que sucede (linha preta pontilhada), referente as respostas dos estudantes do curso de Apicultura do IFRN, Pau dos Ferros-RN, 2018.

As análises estatísticas indicaram uma diferença significativa $(p<0,05)$ no número de questões assinaladas corretamente pelos alunos em ambas as avaliações, rejeitando a hipótese nula (H0). A melhoria do desempenho foi notada em todos os aspectos do diagnóstico estatístico descritivo, visto que houve um aumento significativo da quantidade média, número mínimo e máximo de alunos que responderam corretamente as questões, demonstrado na Tabela 1. 
Tabela 1: Média, número mínimo e máximo de alunos que responderam corretamente o questionário antes e depois da construção e utilização da coleção de abelhas utilizada pelos estudantes do curso de Apicultura do IFRN, Pau dos Ferros-RN, 2018.

\begin{tabular}{c|c|c|c|c|c|c|c}
\hline Variável & N & Média & EP Médio & DesvPad & Mínimo & Mediana & Máximo \\
\hline Antes & 26 & 35,15 & 3,26 & 16,6 & 2 & 34 & 58 \\
Depois & 26 & 56,15 & 2,3 & 11,72 & 25 & 59,5 & 68 \\
\hline
\end{tabular}

Aprender uma nova ciência não é apenas inserir novos conceitos, mas fazer com que os alunos reflitam e criem perspectivas usando os experimentos didáticos como instrumento facilitador na construção de novas ideias (TEIXEIRA, 2014; COSTA et al., 2014; MORAES; JUNIOR, 2015). Na Figura 2, é possível perceber que o resultado das 26 questões, nos diferentes momentos, também indicou uma melhoria significativa no rendimento geral, uma vez que uma maior mediana e um menor desvio padrão do quantitativo de alunos que assinalaram as questões corretas é prova que houve uma consolidação do aprendizado, mostrando que a construção da caixa entomológica como prática de ensino complementar é consistente e pedagogicamente positiva, facilitando o processo de ensino-aprendizagem e promovendo uma melhor relação aluno-professor.

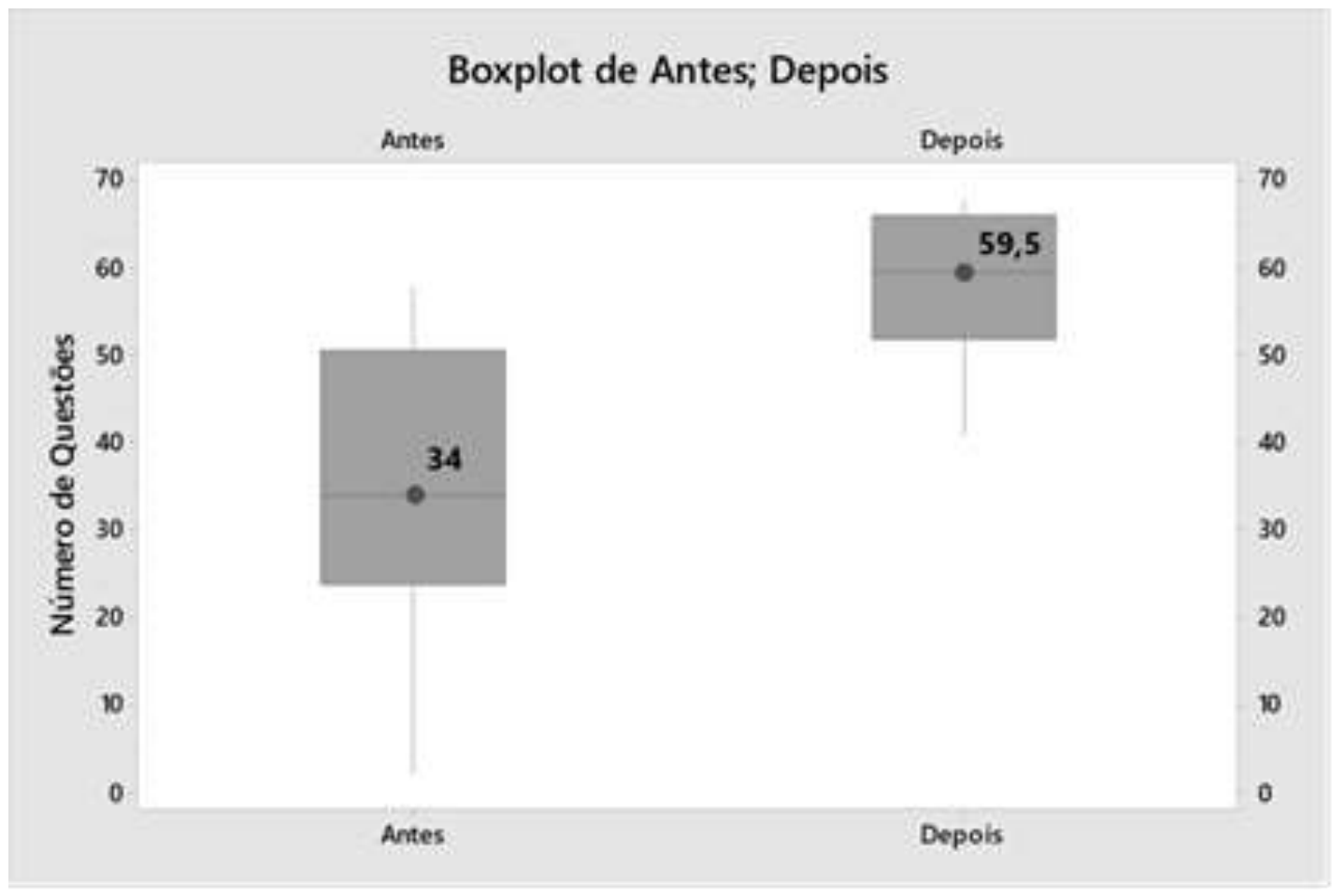

Figura 2: Bloxplot das respostas de antes e depois da coleção de abelhas ser utilizada pelos alunos do curso de Apicultura do IFRN, Pau dos Ferros-RN, 2018.

Em algumas questões aplicadas após a confecção da caixa entomológica, os quantitativos de respostas corretas foi extraordinário, como por exemplo quando questionado "Quais as três partes em que se divide o corpo das abelhas?", apenas $47 \%$ alunos responderam corretamente na primeira avaliação, porém, quando feito o mesmo questionamento logo depois da construção da coleção entomológica, houve um aumento significativo nos valores, que atingiram $100 \%$ de acertos sendo $\mathrm{C}$ a alternativa correta, comprovando a eficácia da ferramenta, como é possível observar na Figura 3. 


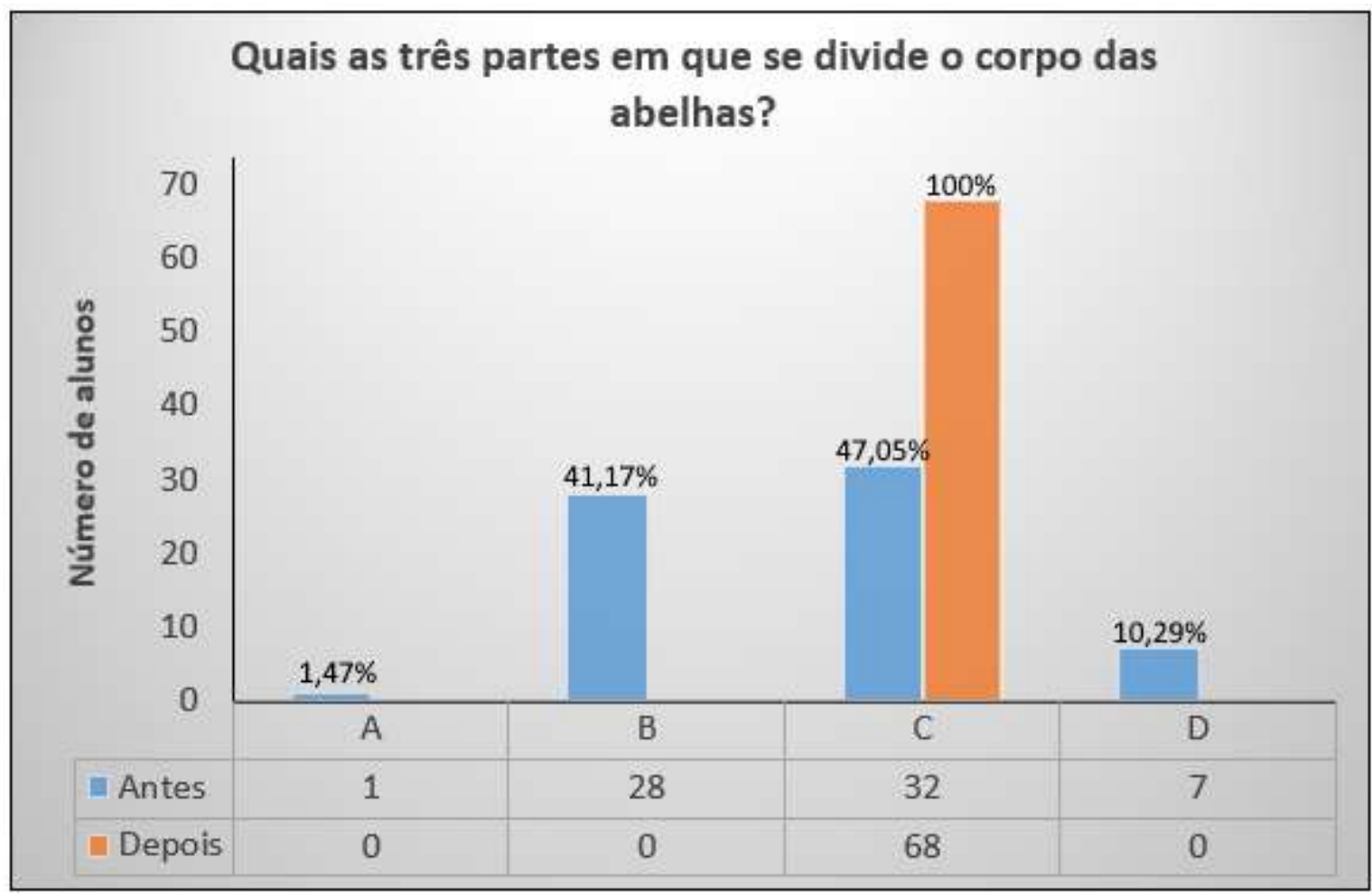

Figura 3: Respostas dos estudantes do curso de Apicultura do IFRN sobre quais as três partes em que se divide o corpo das abelhas, onde A, B, C e D correspondem as alternativas da pergunta, sendo C a alternativa correta. As colunas azuis correspondem as respostas antes da utilização da ferramenta didática e a laranja após a utilização do método de ensino. Pau dos Ferros-RN, 2018.

A vivência com experimentação facilita a fixação de conteúdos relacionados com o objeto de estudo, anulando a ideia de que ferramentas práticas servem apenas como objeto ilustrativo (VIEIRA et al., 2013; SOUZA, 2013; SANTOS; SOUZA, 2016). A Figura 4 representa uma evolução bastante expressiva quanto ao conhecimento internalizado, pois quando se perguntou "Quantos olhos possui uma abelha?", 97\% dos alunos marcaram a alternativa incorreta na primeira etapa, demonstrando ausência de conhecimento relacionado à anatomia do inseto. Já na segunda etapa, 46 alunos marcaram corretamente (Alternativa D), correspondendo a $67 \%$ de acertos, demostrando evolução da aprendizagem após as aulas práticas com a utilização do instrumento didático. Esse resultado pode ser otimizado com a reutilização da coleção entomológica posteriormente para que haja a fixação do conhecimento. 


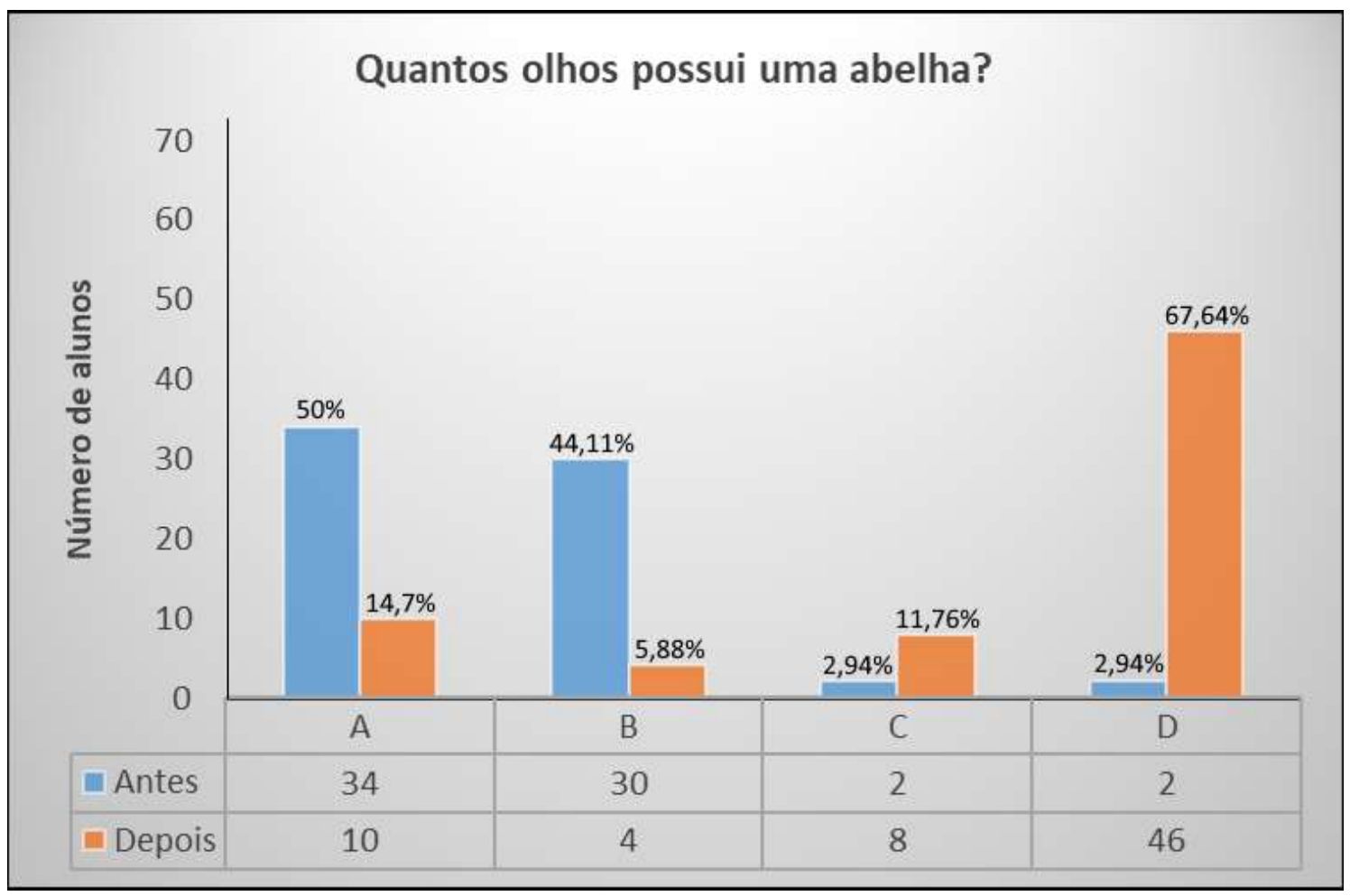

Figura 4: Respostas dos estudantes do curso de Apicultura do IFRN sobre quantos olhos possui uma abelha, onde A, B, C e D correspondem as alternativas da pergunta, sendo as colunas azuis as respostas antes da utilização da ferramenta didática e as laranjas após a utilização do método de ensino. Pau dos Ferros-RN, 2018.

Dentre as 26 questões apresentadas, uma delas consistia na identificação de imagens de abelhas junto com outros insetos. Nesse quesito, houve um aumento significativo na evolução das respostas dos discentes que passaram de $25 \%$ para $64 \%$ de acertos, atestando a eficácia da coleção como objeto facilitador na identificação de abelhas entre outros insetos, tendo em vista a grande dificuldade dos pesquisadores e inclusive apicultores em diferenciar abelhas (FRANCOY; IMPERATRIZ- FONSECA, 2010). Uma das abelhas mais selecionadas nesta questão, além da Apis mellifera, foi a mamangava que pertence ao gênero Xylocopa e é popularmente conhecida como a principal polinizadora do maracujá (Passiflora edulis) e facilmente confundida com besouros (SILVA et al., 2014). Além disso, o tempo médio utilizado para resolução do questionário também variou, passando de 20 (vinte) para 16 (dezesseis) minutos, indicando que os alunos tiveram mais facilidade de responder os questionários após a construção da coleção de abelhas.

Uma das últimas questões da avaliação sugeria a identificação das partes do corpo da abelha Apis mellifera (antenas, cabeça, tórax e abdômen). O índice de acertos aumentou consideravelmente, efetivando a ideia de que as coleções entomológicas, quando associadas ao ensino, facilitam a identificação da morfologia dos insetos. Na tabela 2, é possível observar o percentual de acertos do primeiro questionário, quando comparado com o segundo, na pergunta que diz respeito à identificação de algumas das partes do corpo da abelha Apis mellifera, neste caso o abdome, o tórax, a cabeça e as antenas. 
Tabela 2: Respostas dos estudantes do curso de Apicultura do IFRN sobre a identificação do corpo da abelha Apis mellifera, onde os números 1, 2, 3 e 4 correspondem a quantidade de partes da abelha corretamente assinaladas pelos estudantes. Pau dos Ferros-RN, 2018.

\begin{tabular}{c|c|c}
\hline Quantidade de acertos & Primeira aplicação (\%) & Segunda aplicação (\%) \\
\hline 1 & 7,35 & 0 \\
2 & 29,4 & 2,9 \\
3 & 19,1 & 17,9 \\
4 & 44,1 & 79,4 \\
\hline
\end{tabular}

Portanto, quando avaliada a quantidade máxima de acertos, é notório que houve um considerável aumento quando comparadas as respostas da primeira aplicação do questionário com a segunda, passando de $44,1 \%$ para $79,4 \%$ de acertos. O conhecimento adquirido sobre a morfologia dos insetos, mais especificamente das abelhas, através de aulas práticas é fundamental para os profissionais da agropecuária e áreas afins, evitando práticas de alto impacto negativo na natureza e perda de investimento econômico. As caixas entomológicas são fundamentais para o estudo dos insetos e, quando organizadas, fornecem informações essenciais para vários estudos, como controle de pragas e exigências ecológicas (CAMARGO et al., 2015).

\section{CONCLUSÃO}

É possível perceber que a coleção entomológica possui grande potencial como material didático, visto que os alunos ampliaram consideravelmente sua aprendizagem após a utilização da ferramenta em sala de aula, demostrando que a interação com o material prático facilita a identificação, diferenciação e morfologia de abelhas, potencializando a consolidação do conhecimento.

\section{REFERÊNCIAS}

ALBUQUeRQUE, F. P., MILLÉO, J., de LIMA, J. M. M., de FREITAS BARBOLA, I. (2014). Entomologia no ensino médio técnico agrícola: Uma proposta de trabalho. Revista Eletrônica de Educação, 8(3), 236-250.

ATAIDE, M. C. E. S., da CRUZ SILVA, B. V. (2011). As metodologias de ensino de ciências: contribuições da experimentação e da história e filosofia da ciência. HOLOS, 4, 171-181.

BEZERRA, L., MAUÉS, M. M. (2017). Organização do acervo de Apoidea da coleção entomológica da Embrapa Amazônia Oriental. In Embrapa Amazônia Oriental-Artigo em anais de congresso (ALICE). In: SEMINÁRIO DE INICIAÇÃO CIENTÍFICA DA EMBRAPA AMAZÔNIA ORIENTAL, 21., 2017, Belém, PA. Anais. Belém, PA: Embrapa Amazônia Oriental, 2017.

CAMARGO, A. J. A., de OLIVEIRA, C. M., FRIZZAS, M. R., SONODA, K. C., CORRÊA, D. (2015). Coleções entomológicas: legislação brasileira, coleta, curadoria e taxonomias para as principais ordens. Embrapa Cerrados-Livro científico (ALICE). 118p. 
COSTA, L. M. E., FARIAS, R. A. de M. A., LIMA, S. H. S. (2014). Ciências nos anos iniciais do ensino fundamental: a experimentação como instrumento da prática pedagógica e sua realidade em sala de aula. João Pessoa: UFPB. 62f.; il. Monografia (graduação em Pedagogia) - UFPB/CE.

FRANCOY, T. M., IMPERATRIZ-FONSECA, V. L. (2010). A morfometria geométrica de asas e a identificação automática de espécies de abelhas. Oecologia Australis, 14(1), 317-321.

GUIMARÃES-BRASIL, M. O., SALES, F. A. L., SOUZA, E., BRASIL, D. (2017). CONSTRUÇÃO DE CAIXAS ENTOMOLÓGICAS COMO FERRAMENTA AO ENSINO-APRENDIZAGEM EM CURSOS TÉCNICOS DE AGRÁRIAS. HOLOS, 1, 21-30.

GULLAN, P. J., CRANSTON, P. S. (2010). The insects: an outline of entomology. John Wiley \& Sons. 4th ed.; Wiley: Hoboken, NJ, USA; Volume 14.

MORAES, J. U. P., JUNIOR, R. S. S. (2015). Experimentos didáticos no ensino de física com foco na aprendizagem significativa. Lat. Am. J. Phys. Educ. Vol, 9(2), 2504-1.

PEREIRA, A. C. (2016). O uso de coleções entomológicas como ferramenta de ensino na educação básica no Brasil. Revista da SBEnBio, n. 9, p. 4437- 4448.

SANTOS, D. C. de J., SOUTO, L de S. (2011). Coleção entomológica como ferramenta facilitadora para a aprendizagem de Ciências no ensino fundamental. Scientia plena, 7(5), 1-8.

SANTOS, G. G., SOUZA, D N. (2016). Experimentação real versus experimentação ideal no ensino de ciências e a prática do pensamento crítico. Scientia Plena, 12(11), 1-11.

SILVA, C. I. et al. (2014). Guia Ilustrado de Abelhas Polinizadoras no Brasil. São Paulo, Instituto de Estudos Avançados da USP/ Ministério do Meio Ambiente. 50 p.

SILVA, T. M. D. S. (2018). O USO DE COLEÇÕES ENTOMOLÓGICAS COMO FERRAMENTA NO ENSINO DE BIOLOGIA. Encontro Internacional de Formação de Professores e Fórum Permanente de Inovação Educacional, 11(1).

SOUSA-LOPES, B. de. (2017). Sobre o uso de uma coleção entomológica como ferramenta didática no ensino médio noturno e a percepção de fatores que influenciam o baixo rendimento escolar. Experiências em Ensino de Ciências, São Paulo, v. 12, n. 8, p.250-260.

SOUZA, A. C. (2013). A experimentação no ensino de ciências: importância das aulas práticas no processo de ensino aprendizagem. Monografia de Especialização da UTFPR. Medianeira.

TEIXEIRA, L. P. (2014). Experimentação investigativa em ciências e a formação do conceito de germinação. Dissertação de Mestrado em Educação em Ciências e Matemática. 2014. 151 f.: il., figs.

VIEIRA, B. D. C. R., LORENZONI, L. S., GOBBO, S. D. A., BRECHIANI, M. C. M., SOUZA, M. H. A. (2013). A importância da experimentação em Ciências para a construção do conhecimento no ensino fundamental. Enciclopedia Biosfera, 9(16), 2276-2285. 